Einführung in die germanistische Linguistik 
Jörg Meibauer/Ulrike Demske/Jochen Geilfuß-Wolfgang/ Jürgen Pafel/Karl Heinz Ramers/Monika Rothweiler/ Markus Steinbach

\section{Einführung in die germanistische Linguistik}




\section{Die Autorinnen und Autoren}

Jörg Meibauer, Professor für Sprachwissenschaft des Deutschen an der Universität Mainz;

Ulrike Demske, Professorin für Germanistische Linguistik an der Universität des Saarlandes;

Jochen Geilfuß-Wolfgang, Wissenschaftlicher Assistent am Institut für Germanistik der Universität Leipzig;

Jürgen Pafel, Professor für Germanistische Linguistik an der Universität Stuttgart;

Karl Heinz Ramers, Privatdozent für Germanistische Linguistik an der Universität Stuttgart;

Monika Rothweiler, Professorin für Angewandte Sprachwissenschaft und Phonetik an der Universität Hamburg;

Markus Steinbach, Akademischer Rat am Deutschen Institut der Universität Mainz.

Die Deutsche Bibliothek - CIP-Einheitsaufnahme

Einführung in die germanistische Linguistik /

Jörg Meibauer ...

- Stuttgart ; Weimar : Metzler, 2002

ISBN 978-3-476-01851-9

ISBN 978-3-476-01851-9

ISBN 978-3-476-02789-4 (eBook)

DOI 10.1007/978-3-476-02789-4

Dieses Werk einschließlich aller seiner Teile ist urheberrechtlich geschützt. Jede Verwertung außerhalb der engen Grenzen des Urheberrechtsgesetzes ist ohne Zustimmung des Verlages unzulässig und strafbar. Das gilt insbesondere für Vervielfältigungen, Übersetzungen, Mikroverfilmungen und die Einspeicherung und Verarbeitung in elektronischen Systemen.

(C) 2002 Springer-Verlag GmbH Deutschland

Ursprünglich erschienen bei J. B. Metzlersche Verlagsbuchhandlung

und Carl Ernst Poeschel Verlag GmbH in Stuttgart 2002

www.metzlerverlag.de

info@metzlerverlag.de 


\section{VORWORT}

Dieses Lehrbuch ist eine Einführung in die germanistische Linguistik, die während des ganzen Studiums benutzt werden kann. In der Einleitung wird der Gegenstand `Sprache als ein soziales und historisches Phänomen und zugleich als eine biologisch und kognitiv fundierte Fähigkeit des Menschen charakterisiert. In den fünf folgenden Kapiteln werden die linguistischen Kerngebiete 'Lexikon und Morphologie, , Phonologie`, 'Syntax‘, 'Semantik` und 'Pragmatik` behandelt. Diese Kapitel erläutern jeweils sprachwissenschaftliche Grundbegriffe, illustrieren sie an Beispielen aus dem Deutschen und geben einen Einblick in die linguistische Theoriebildung. Daran anschließend werden mit dem kindlichen Spracherwerb und dem Sprachwandel zwei Gebiete vorgestellt, die von großer Bedeutung für ein tieferes Verständnis der menschlichen Sprache sind. In alle Kapitel sind Übungen integriert, die die Kontrolle des Verständnisses erleichtern und zu selbstständiger Analyse und kritischer Reflexion anleiten. Musterlösungen zu diesen Übungen finden Sie unter www.egli-online.de.

$\mathrm{Zu}$ jedem Kapitel wird auf ausgewählte Fachliteratur verwiesen. Darüber hinaus gibt es eine allgemeine Bibliographie mit gezielten Hinweisen auf andere Einführungen, auf Lexika und Handbücher, auf Grammatiken, Wörterbücher und Fachzeitschriften und ein kleines Verzeichnis ausgewählter, linguistisch interessanter Internetadressen. Ein umfangreiches Glossar mit knappen Erläuterungen der wichtigsten Fachtermini und ein Sachregister schließen dieses Lehrbuch ab.

Insgesamt ist unser Ziel, die Anfängerin und den Anfänger für das Studium der germanistischen Linguistik zu motivieren und fortgeschrittene Studierende an den aktuellen Stand der Disziplin heranzuführen, wobei wir uns an der internationalen Diskussion in der Linguistik orientiert haben, ohne dass einseitig eine bestimmte theoretische Richtung bevorzugt wird. Die Auswahl der Gegenstände in den einzelnen Kapiteln erfolgte aufgrund langjähriger Lehrerfahrung; Vollständigkeit konnte jedoch allein schon aus Platzgründen nicht angestrebt werden. Die einzelnen Kapitel, in denen immer auch grundlegendes Wissen über die deutsche Grammatik behandelt wird, sind in intensiver Diskussion aufeinander abgestimmt worden. Sie können unabhängig voneinander gelesen werden, wobei sich das Lehrbuch zum Selbststudium genauso eignet wie als Kurslektüre.

Wir danken den folgenden Personen, die uns bei der Arbeit geholfen haben: Anne-Katrin Heymann, Caroline Mannweiler, Juliane Möck, Ingo Reich, Carmen Scherer, Melani Vukosav, Tanja Werner und last but not least Ute Hechtfischer vom Metzler Verlag.

Im Juni 2002

Die Autor/innen 


\section{INHALT}

1. Einleitung (Jörg Meibauer/Markus Steinbach) ........................................... 1

1.1 Sprache in Literatur und Alltag ............................................................... 1

1.2 Sprache als soziales Phänomen ……………………................................. 3

1.3 Sprache als historisches Phänomen .......................................................... 5

1.4 Sprache als biologisches Phänomen …………….................................... 6

1.5 Sprache als kognitives Phänomen ........................................................ 8

1.6 Linguistik als Geisteswissenschaft ...................................................... 10

1.7 Zum Nutzen der Linguistik ............................................................... 11

1.8 Zum Inhalt des Buchs ..................................................................... 12

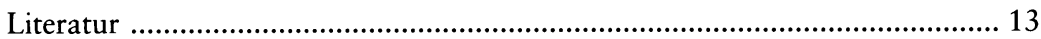

2. Lexikon und Morphologie (Jörg Meibauer) ............................................ 15

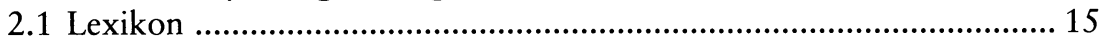

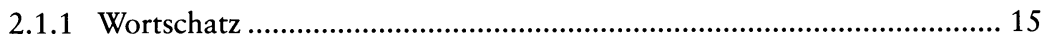

2.1.2 Wörterbuch und Lexikon ...................................................................... 15

2.1.3 Zur Definition des Worts ...................................................................... 17

2.1.4 Eigenschaften des Lexikons ............................................................... 18

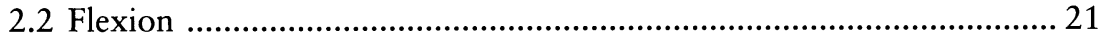

2.2.1 Wort und Wortform .......................................................................... 21

2.2.2 Zur nominalen Flexion ..................................................................... 23

2.2.3 Zur Flexion des Verbs .................................................................... 26

2.3 Grundlagen der Wortbildung .............................................................. 29

2.3.1 Morphologische Grundbegriffe ......................................................... 29

2.3.2 Typen der Wortbildung ……………………………………………. 32

2.3.3 Wortstruktur ………………………………………………………. 34

2.3.4 Wortbildungsregeln ......................................................................... 36

2.3.5 Der Kopfbegriff in der Wortbildung ...................................................... 38

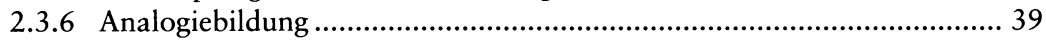

2.4 Wortbildung im Lexikon....................................................................... 40

2.4.1 Zum Lexikonbegriff .............................................................................. 40

2.4.2 Lexikoneinträge ………………………………………………….... 41

2.4.3 Blockierung ……………………………………………………..... 43

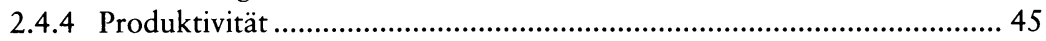

2.5 Komposition .......................................................................................... 48

2.5.1 Haupttypen der Komposition ............................................................ 48

2.5.2 Fugenelemente …………………………………….......................... 49

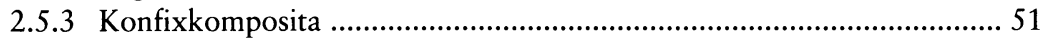

2.5.4 Interpretation von $\mathrm{N}+\mathrm{N}-$ Komposita ………………………………..... 52

2.5.5 Phrasenkompositum ......................................................................... 54

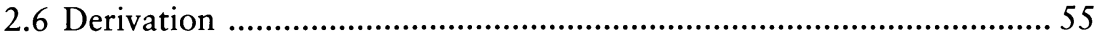

2.6.1 Haupttypen der Derivation ………………………………………..... 55

2.6.2 Präfix- und Partikelverben ................................................................... 59 
VIII Inhalt

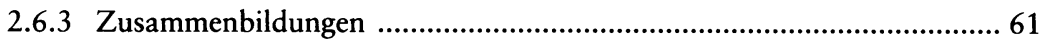

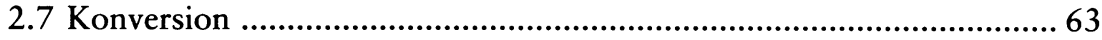

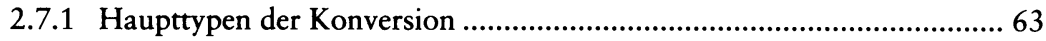

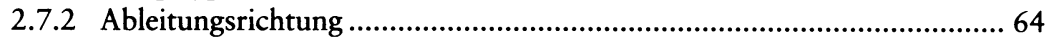

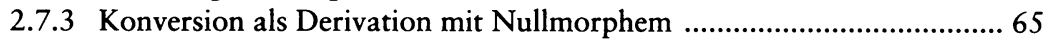

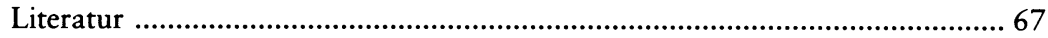

3. Phonologie (Karl Heinz Ramers) .......................................................... 70

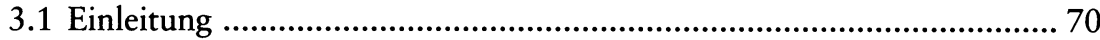

3.2 Phonetische Grundlagen ............................................................. 72

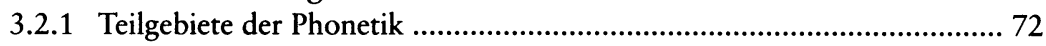

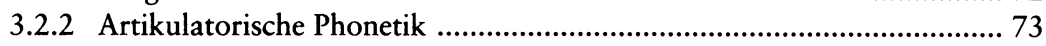

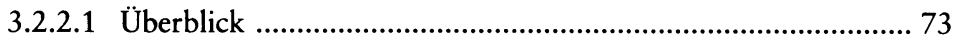

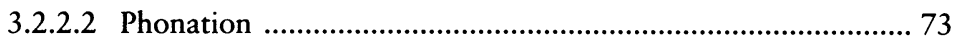

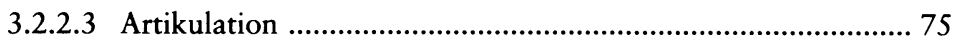

3.3 Segmentale Phonologie ........................................................ 81

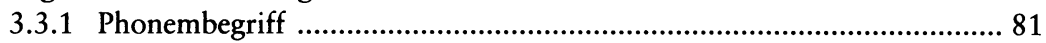

3.3.2 Allophone und Variationsarten .................................................... 83

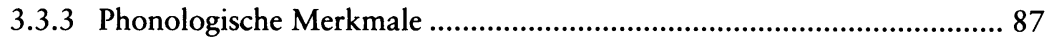

3.3.4 Phonologische Prozesse und Regeln ................................................ 94

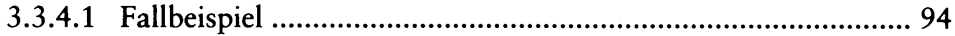

3.3.4.2 Bedingungen für phonologische Prozesse .............................. 95

3.3.4.3 Typen phonologischer Prozesse ............................................... 97

3.3.4.4 Phonologische Regeln ........................................................... 99

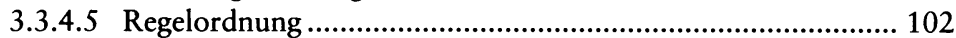

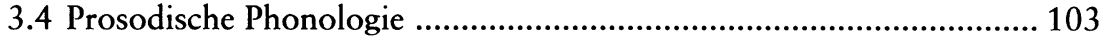

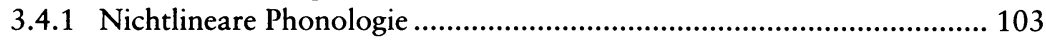

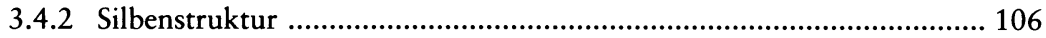

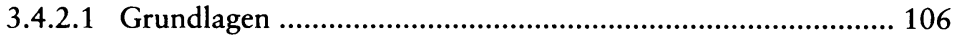

3.4.2.2 Die Sonoritätshierarchie ...................................................... 108

3.4.2.3 Quantitätsstrukturen im CV-Modell der Silbe ....................... 111

3.4.2.4 Konstituentenstruktur der Silbe ........................................... 113

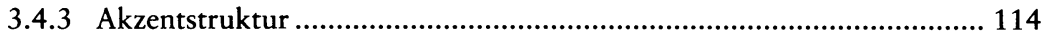

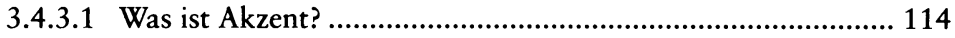

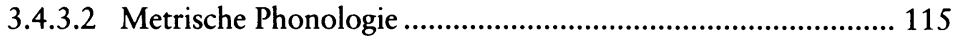

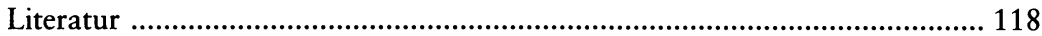

4. Syntax (Jochen Geilfuß-Wolfgang) ..................................................... 121

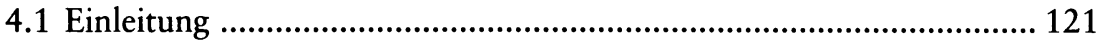

4.2 Topologische Felder ............................................................ 121

4.3 Konstituentenstruktur .............................................................. 125

4.4 Syntaktische Kategorien ........................................................ 129

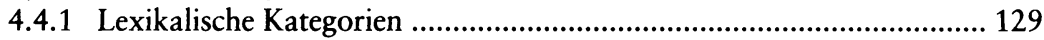

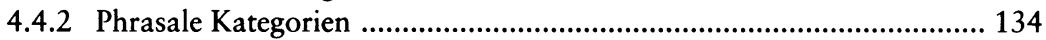

4.5 Eingebettete Sätze .................................................................... 138

4.5.1 Zur formalen Klassifizierung der eingebetteten Sätze ........................ 139

4.5.2 Eingebettete Sätze und topologische Felder ...................................... 141

4.6 Zur Struktur von infiniten Sätzen, w-Sätzen und Relativsätzen ......... 143 
4.7 Zur Struktur von V1- und V2-Sätzen ......................................... 145

4.8 Argumentstruktur ................................................................... 148

4.8.1 Argumente und ihre syntaktischen Realisierungen ........................... 148

4.8.2 Semantische Rollen ..................................................................... 150

4.9 Syntaktische Funktionen ........................................................ 152

4.9.1 Syntaktische Funktionen und syntaktische Kategorien ....................... 152

4.9.2 Warum syntaktische Funktionen? ................................................... 153

4.9.3 Welche syntaktischen Funktionen gibt es? ........................................ 154

4.9.4 Syntaktische Funktionen und Argumentstruktur .............................. 159

Literatur ......................................................................................... 160

5. Semantik (Markus Steinbach) ............................................................. 162

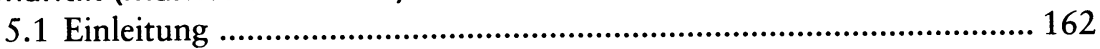

5.1.1 Produktivität ................................................................................ 162

5.1.2 Gleiches, Verschiedenes und Mehrdeutiges ........................................ 164

5.1.3 Wortsemantik, Satzsemantik und Diskurssemantik ............................ 167

5.1.4 Was ist die Bedeutung sprachlicher Ausdrücke? ................................ 169

5.1.5 Satzbedeutung, Äußerungsbedeutung und Sprecherbedeutung ............ 173

5.2 Lexikalische Semantik ............................................................. 176

5.2.1 Intensionen, Extensionen und Referenzen ....................................... 176

5.2.2 Semantische Relationen ............................................................... 180

5.2.3 Lexikalische Dekomposition ......................................................... 184

5.2 .4 Prototypen .................................................................................. 188

5.2.5 Polysemie und Unterspezifikation ................................................... 190

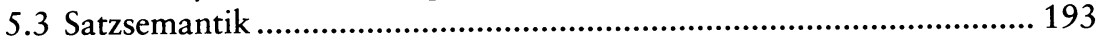

5.3.1 Verben, Aktionsarten und semantische Rollen ................................. 193

5.3.2 Sätze, Situationen und Wahrheitswerte ............................................ 198

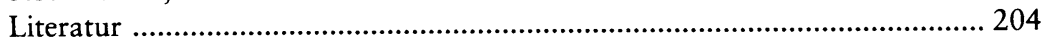

6. Pragmatik (Jürgen Pafel) ..................................................................... 208

6.1 Einleitung .............................................................................. 208

6.2 Deixis und Anapher ................................................................ 208

6.2.1 Arten der Deixis ........................................................................... 208

6.2.2 Anaphern .................................................................................. 211

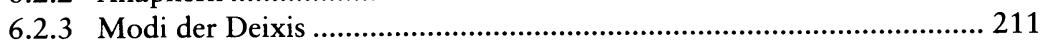

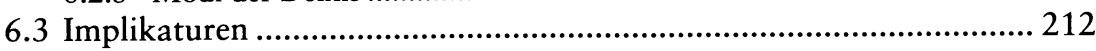

6.3.1 Was sind Implikaturen? ................................................................. 212

6.3.2 Kooperationsprinzip und Konversationsmaximen ............................. 214

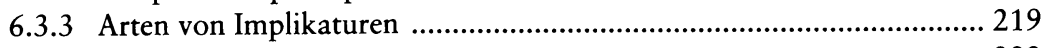

6.3.4 Was auf Grice folgte ..................................................................... 222

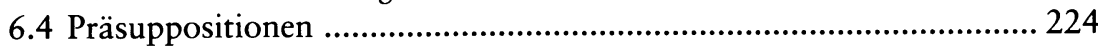

6.4.1 Was sind Präsuppositionen? ........................................................... 224

6.4.2 Suspendierbarkeit und Projektionsproblem ...................................... 226

6.4.3 Präsupposition und Implikatur ........................................................ 227

6.5 Sprechakte ........................................................................ 228

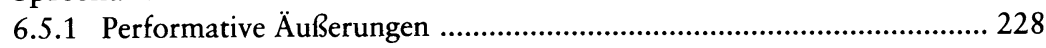

6.5.2 Explizite und implizite Performative ................................................ 230

6.5.3 Aufbau von Sprechakten ................................................................ 233 


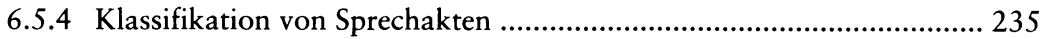

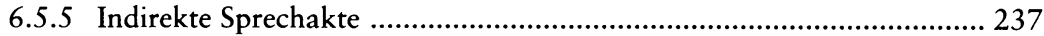

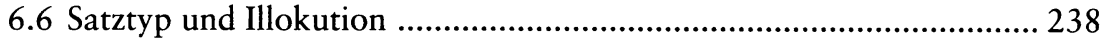

6.7 Fokus-Hintergrund-Gliederung ............................................... 240

6.8 Konversationsstruktur ............................................................ 243

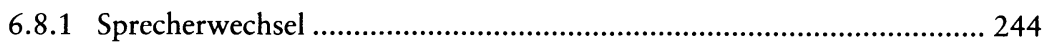

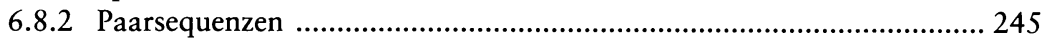

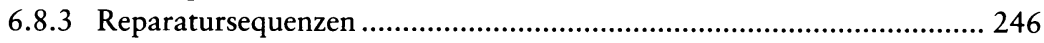

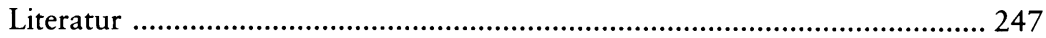

7. Spracherwerb (Monika Rothweiler) ....................................................... 251

7.1 Was versteht man unter Spracherwerb? ........................................ 251

7.2 Der Verlauf des Spracherwerbs .................................................. 255

7.2.1 Sprachwahrnehmung und früher Lauterwerb ................................ 255

7.2.1.1 Sprachwahrnehmung im ersten Lebensjahr ......................... 255

7.2.1.2 Vorsprachliche Lautproduktion .......................................... 257

7.2.2 Einige Aspekte des phonologischen Erwerbs ..................................... 259

7.2.2.1 Die Phase der ersten 50 Wörter ........................................... 259

7.2.2.2 Phonologischer Erwerb und phonologische Prozesse ............. 261

7.2.3 Der Aufbau des kindlichen Lexikons .................................................. 264

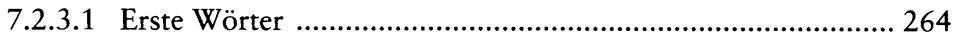

7.2.3.2 Der weitere Ausbau des kindlichen Lexikons ........................ 265

7.2.3.3 Lexikalische Erwerbsstrategien .......................................... 266

7.2.3.4 Wörter und ihre Bedeutungen ............................................. 268

7.2.4 Der Erwerb von Wortbildungsmustern ............................................. 270

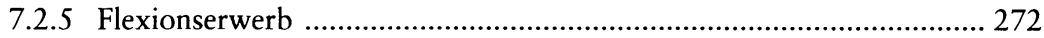

7.2.6 Ein kurzer Einblick in den Syntaxerwerb ......................................... 276

7.2.6.1 Beginn des Syntaxerwerbs .................................................. 277

7.2.6.2 Der Erwerb der Verbstellung im Deutschen .......................... 278

7.2.7 Spracherwerb und Pragmatik ............................................................. 282

7.3 Erklärungsansätze in der Spracherwerbsforschung ........................ 284

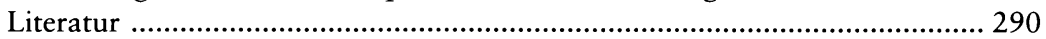

8. Sprachwandel (Ulrike Demske) …………………................................ 294

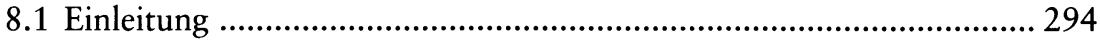

8.2 Lautwandel ................................................................................. 297

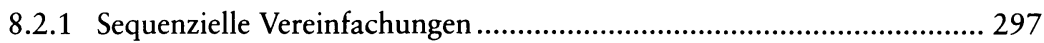

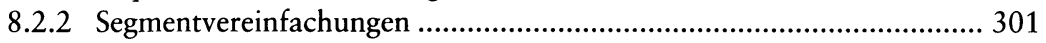

8.2.3 Die Interpretation lautlicher Veränderungen ................................... 301

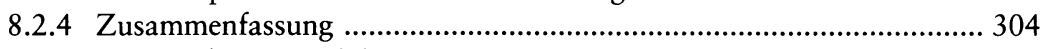

8.3 Morphologischer Wandel ......................................................... 304

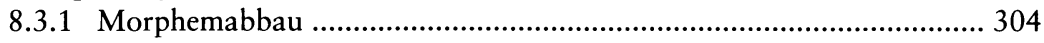

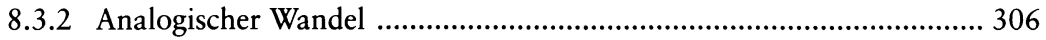

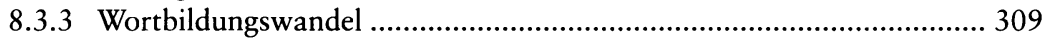

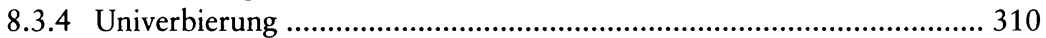

8.3.5 Natürlicher morphologischer Wandel .......................................... 311

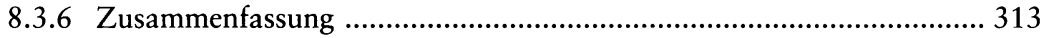

8.4 Syntaktischer Wandel ............................................................ 314 
Inhalt XI

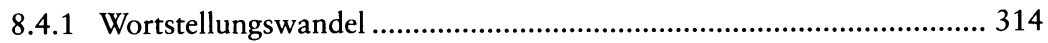

8.4.2 Wandel der Selektionsbeziehung ....................................................... 316

8.4.3 Das Prinzip der natürlichen Serialisierung ......................................... 318

8.4.4 Syntaktischer Wandel und Flexionsmorphologie ................................ 319

8.5 Semantischer Wandel .............................................................. 322

8.5.1 Bedeutungsverengung vs. Bedeutungserweiterung ............................. 322

8.5.2 Bedeutungsverbesserung vs. Bedeutungsverschlechterung .................. 323

8.5.3 Bedeutungsübertragung und Bedeutungsverschiebung ........................ 324

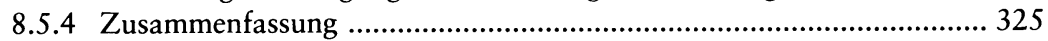

8.6 Sprachwandel durch Sprachkontakt .......................................... 326

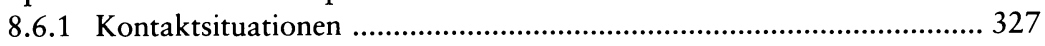

8.6.2 Interferenzen in der deutschen Sprachgeschichte .............................. 327

8.6.3 Zusammenfassung ........................................................................ 332

8.7 Die Ausbreitung von Sprachwandel ........................................... 333

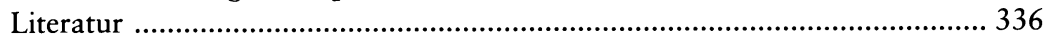

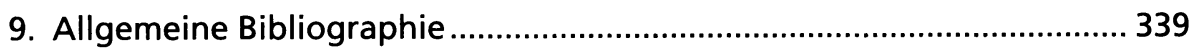

9.1 Einführungen in die Linguistik .................................................. 339

9.2 Nachschlagewerke .................................................................. 340

9.3 Handbücher .............................................................................. 340

9.4 Grammatiken des Deutschen ..................................................... 343

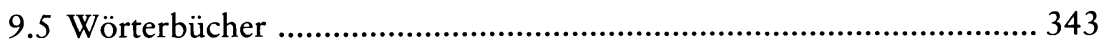

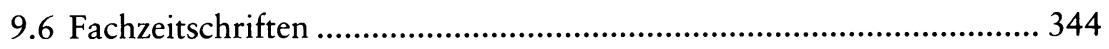

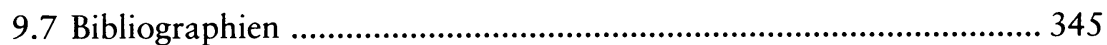

9.8 Ausgewählte Internetadressen ............................................ 345

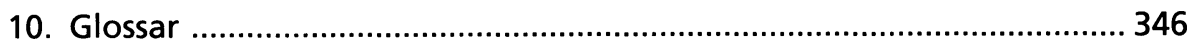

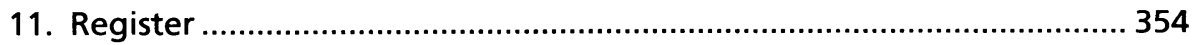

